\title{
Review of Trajectories for the Automated Paint Application, Containment, and Treatment System
}

Richard J. Norcross

U.S. DEPARTMENT OF COMMERCE Technology Administration National Institute of Standards and Technology

Intelligent Systems Division

100 Bureau Drive, Stop 8230

Gaithersburg, MD 20899-8230 



\section{Review of Trajectories for the Automated Paint Application, Containment, and Treatment System}

Richard J. Norcross

U.S. DEPARTMENT OF COMMERCE Technology Administration National institute of Standards and Technology

Intelligent Systems Division

100 Bureau Drive, Stop 8230

Gaithersburg, MD 20899-8230

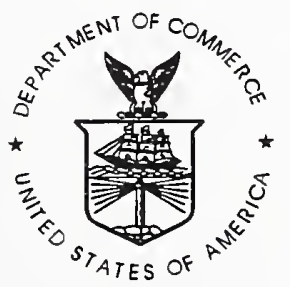

U.S. DEPARTMENT OF COMMERCE William M. Daley, Secretary

TECHNOLOGY ADMINISTRATION Gary R. Bachula, Acting Under Secretary for Technology

NATIONAL INSTITUTE OF STANDARDS

AND TECHNOLOGY

Raymond G. Kammer, Director 



\title{
Review of Trajectories for the
}

\section{Automated Paint Application, Containment, and Treatment System}

\author{
Richard J. Norcross
}

\author{
Intelligent Systems Division \\ National Institute of Standards and Technology \\ Technology Administration \\ U.S. Department of Commerce \\ Gaithersburg, Maryland 20899
}

April 16, 1998 


\section{Disclaimer}

No approval or endorsement of any commercial product by the National Institute of Standards and Technology is intended or implied. Certain commercial equipment, instruments, or materials are identified in this report to facilitate understanding. Such identification does not imply recommendation or endorsement by the National Institute of Standards and Technology, nor does it imply that the materials or equipment identified are necessarily the best available for the purpose.

\section{Copyright}

This publication was prepared by United States Government employees as part of their official duties and is, therefore, a work of the U.S. Government and not subject to copyright.

\section{Acknowledgement}

This report is partial fulfillment of sub-contract AM 02-9802001 with AmDyne Corporation of Millersville, Maryland. 


\begin{abstract}
The Carderock Division of the Naval Surface Warfare Center is developing the Automated Paint Application, Containment, and Treatment System (APACTS) to apply anticorrosive and anti-fouling paints in an environmentally sound manner. APACTS' motion system employs three manipulators; a self-propelled base, a long reach maxi-manipulator, and a quick response mini-manipulator. This report reviews possible APACTS motion trajectories and identifies the interactions between the trajectory and the performance requirements of other system components. The report evaluates the impact of four trajectories on the complexity of the manipulator, the burden imposed on other APACTS components, and the quality of the paint coat. The comparison computations use approximate values regarding paint application and maxi-manipulator performance that should be accurately measured prior to the final decisions.
\end{abstract}

Review of Trajectories for the Automated Paint Application, Containment, and Treatment System 


\section{Table of Contents}

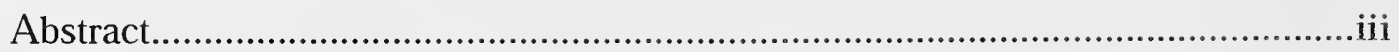

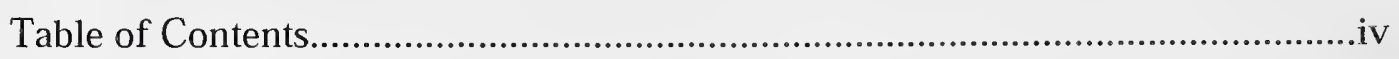

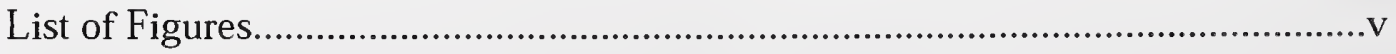

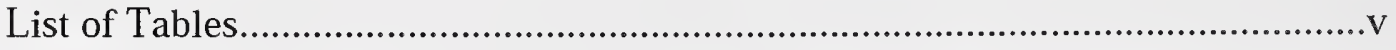

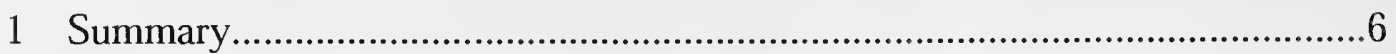

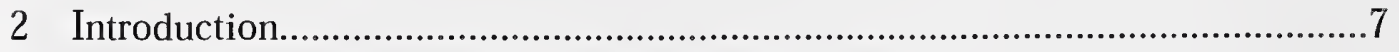

3 Methods, Assumptions, and Procedures.........................................................

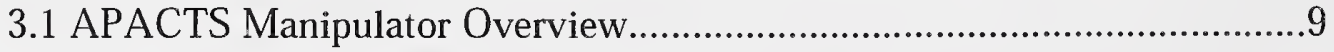

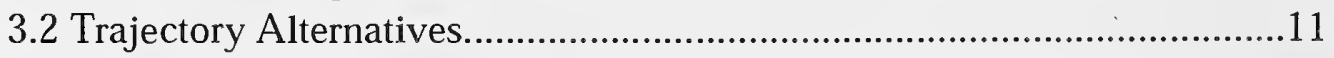

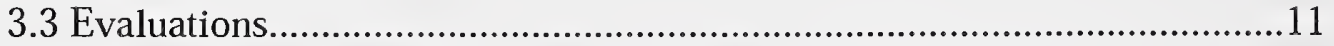

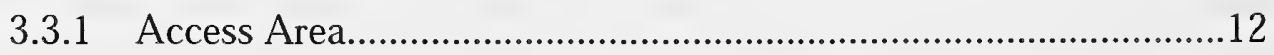

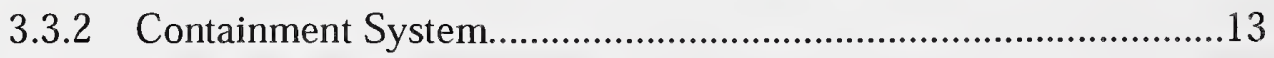

3.3.3 Coating Thickness Estimation......................................................13

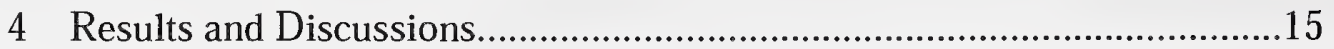

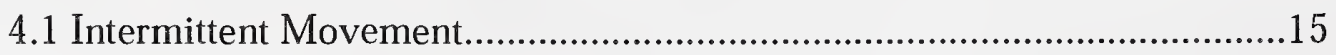

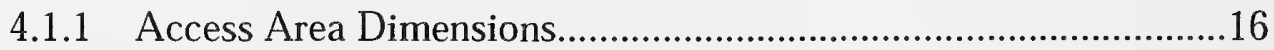

4.1.2 Capture and Treatment Capacity..................................................17

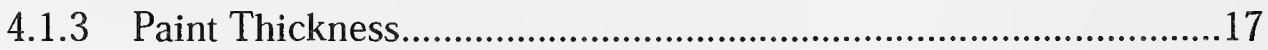

4.2 Perpendicular Nozzles.........................................................................18

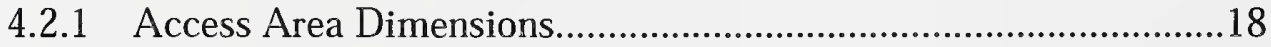

4.2.2 Capture and Treatment Capacity...................................................18

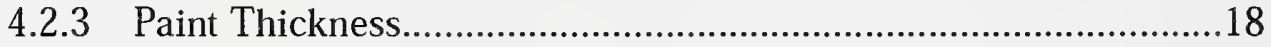

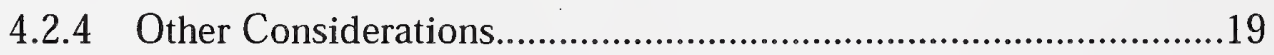

4.3 Zigzag..................................................................................................19

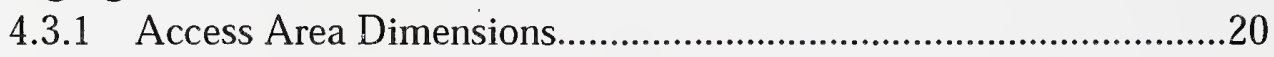

4.3.2 Capture and Treatment Capacity..................................................21

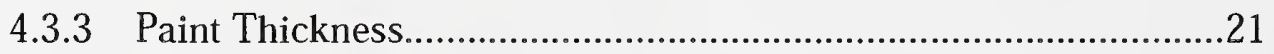

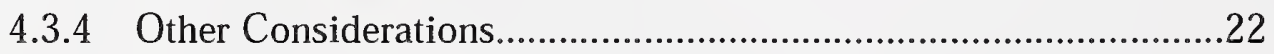

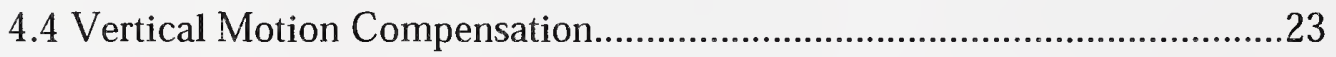

4.4.1 Trajectory Description...............................................................23

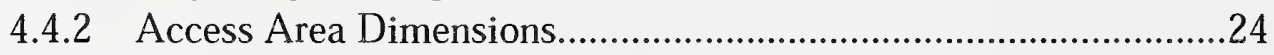

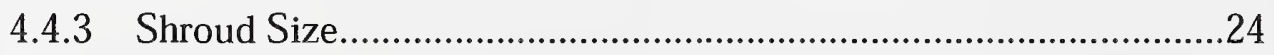

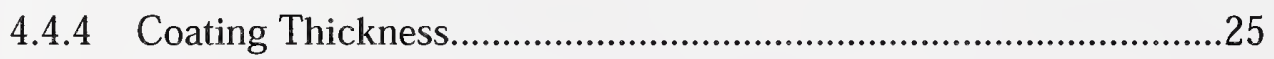

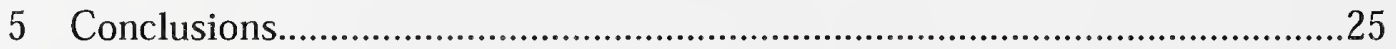

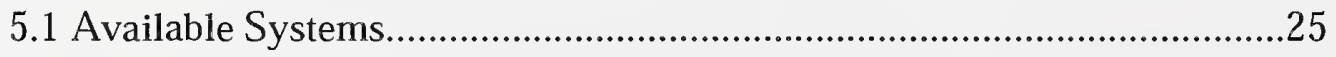

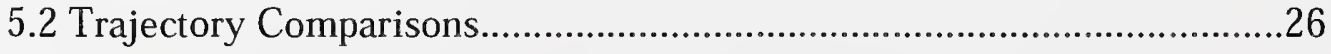

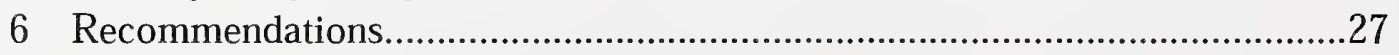

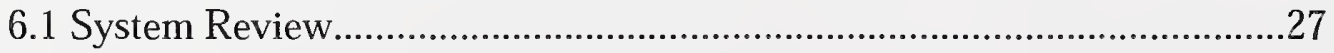

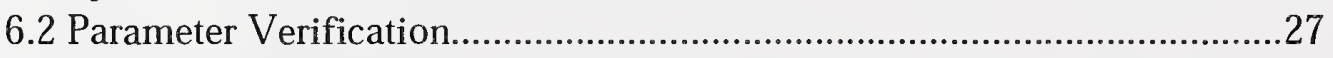

Review of Trajectories for the Automated Paint Application, Containment, and Treatment System 


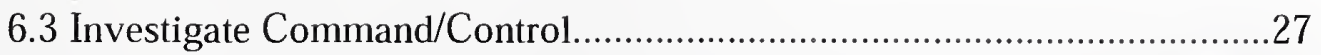

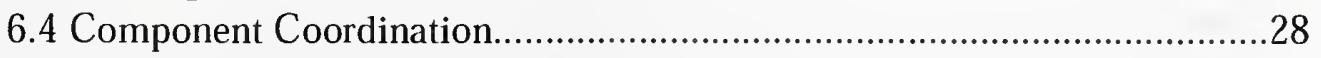

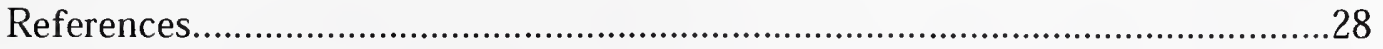

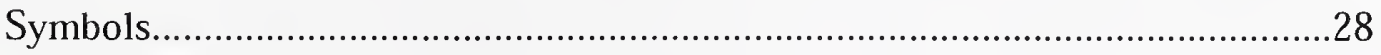

\section{List of Figures}

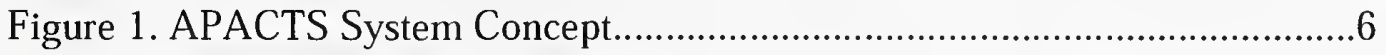

Figure 2. Paint Spray Distribution from Air Paint Gun..........................................15

Figure 3. Zigzag Trajectory..........................................................................20

Figure 4. Zigzag Trajectory Paint Distribution....................................................22

Figure 5. Zigzag Trajectory Paint Distribution with Noise.................................23

Figure 6. Head Trajectories; Relative to (a) Manipulator, (b) Ship........................24

\section{List of Tables}

Table 1. Comparison Values..................................................................................12

Table 2. Comparison Summary..........................................................................22 


\section{Summary}

The Carderock Division of the Naval Surface Warfare Center is developing the Automated Paint Application, Containment, and Treatment System (APACTS) to apply anticorrosive and anti-fouling paints onto Navy ship hulls in an environmentally sound manner. This report reviews four motion-trajectories (a sequence of points traced by a manipulator to accomplish a task) to identify the effects of the interaction between the trajectory and the APACTS' system production requirements. The report also evaluates the trajectory's impact on the complexity of the manipulator, the burden imposed on other APACTS components, and the consistency of the paint coat's thickness.

\section{Figure 1. APACTS System Concept}

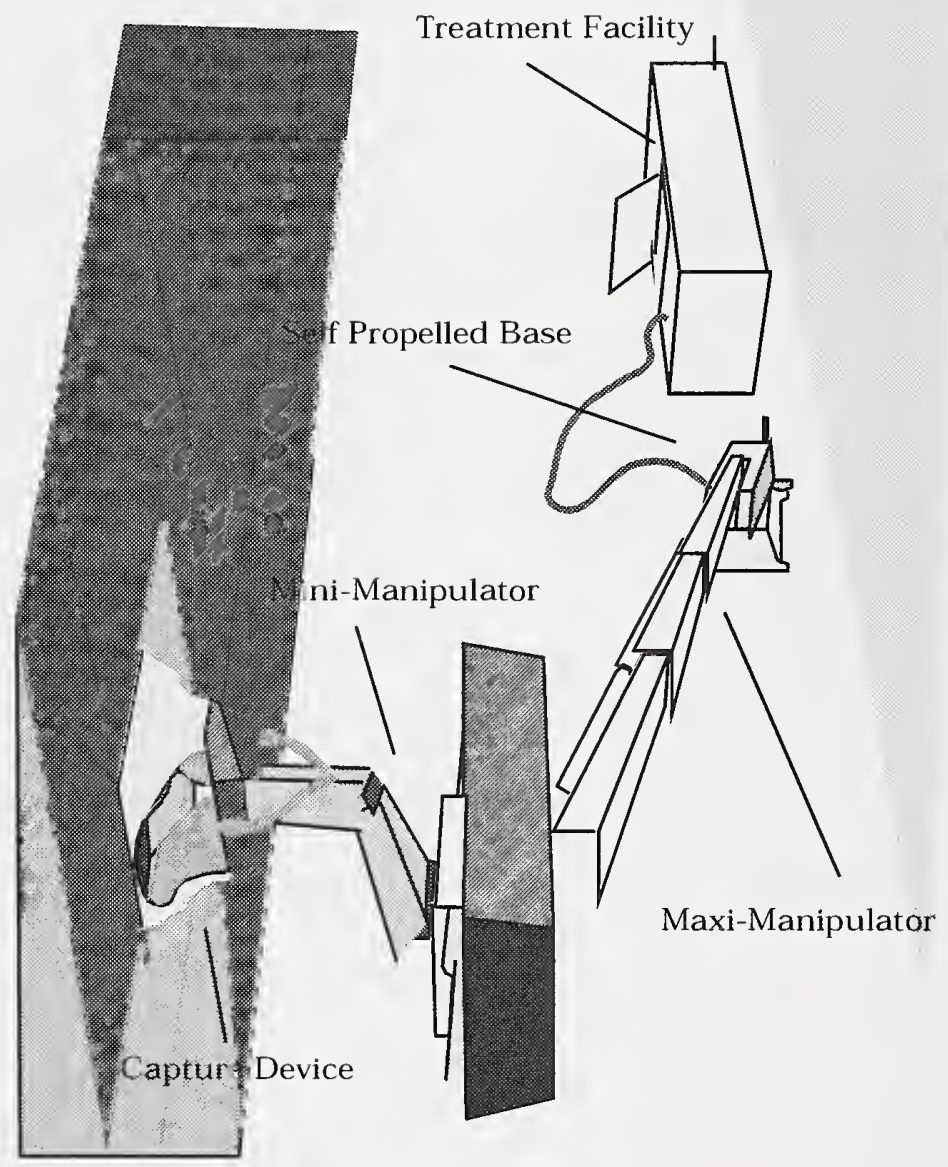

APACTS' motion system (shown in Figure 1) employs three manipulators; a selfpropelled base, a long reach maxi-manipulator, and a quick response mini-manipulator. The combined motions of the maxi and mini-manipulators form the system trajectory. 
This report discusses four possible APACTS trajectories; intermittent movement, perpendicular spray, zigzag, and vertical compensation. An intermittent movement trajectory separates the two manipulators' motions. This trajectory simplifies the control of the manipulators but generates large non-productive periods that require an enormous mini-manipulator to achieve the desired production rates. The perpendicular spray trajectory combines constant maxi-manipulator motion with nozzles mounted perpendicular to that motion. The trajectory simplifies the mini-manipulator design but requires nozzles that will atomize properly without clogging at low flow rates and requires a larger capture and treatment systems. When the nozzles move at a high speed and travel perpendicular to the maxi-manipulator motion, the result is a zigzag trajectory which lays several, very thin paint layers and generates the most even paint coat but requires very fine paint atomization. The vertical compensation trajectory has a constant maxi-manipulator motion and a mini-manipulator motion that combines a perpendicular motion with a motion opposite to the maxi-manipulator's movement. With this trajectory, a conventional nozzle can paint a series of simple stripes with a slightly more complex mini-manipulator.

The trajectory comparisons use unquantified values for the APACTS paint application system and uncertain performance specifications for the maxi-manipulator. The assumed values are derived from operator experience and preliminary experiments. While the values are reasonable, trajectory selection and subsequent manipulator design should follow experiments to quantify those values.

\section{Introduction}

To guard against the harshness of the sea, ship hulls are covered with various paints that must be periodically replaced to maintain their effectiveness. Paint application exposes three significant problem areas. First, manual application produces irregular coverage, leaving some surfaces inadequately protected while wasting money through the deposition of excessive paint on adjacent surfaces. Second, the current paint systems have insufficient service life to fulfill all of the Navy's operational needs. Third, a portion of the paint spray, called the overspray, flows past the surface and falls onto the drydock and surrounding areas. The overspray may contain contaminants such as; heavy metals (e.g., copper and zinc), hazardous air pollutants (HAPs), and volatile organic compounds (VOCs). The airborne overspray exposes shipyard personnel to undesirable and unacceptable risks, while the heavy metal particles deposited in the drydock subsequently migrate to the harbor causing severe and widespread environmental damage.

The Carderock Division, Naval Surface Warfare Center, (CD-NSWC) conducts research and development leading to fleet implementation of materials, processes, and equipment that enable Navy ships and shipyards to better fulfill the Navy's missions. The Paints and Processes Branch (Code 641) advances coating and preservation technologies that 
result in increased performance of Navy coatings and improved material readiness and overall operational performance of Navy ships. The Pollution Prevention and Material Safety Branch (Code 632) provides the Navy with the technical expertise to solve existing and emerging waste management problems to ensure the Navy is environmentally responsible. Pursuant to these responsibilities, CD-NSWC is developing the Automated Paint Application, Containment, and Treatment System (APACTS) to apply advanced coatings, to improve paint application, and to significantly reduce particulate discharge from the painting operation.

APACTS's primary components are the delivery system, the containment system, the treatment system, and the manipulation system. The delivery system consists of a paint mixer, strainer, sprayer, nozzle, and associated equipment. The containment system surrounds the paint sprayer and includes a capture shroud, recovery vacuum, hoses, and controls. The treatment system includes waste transport, waste isolation, filter elements, and their support equipment. The manipulation system consists of those devices that move the sprayer and containment shroud. The components complement each other to produce an effective, economic, and environmentally sound system.

The Intelligent Systems Division of the National Institute of Standards and Technology (NIST-ISD) supports APACTS development through investigation of new and existing technologies to carry, maneuver, and manipulate the APACTS sprayer and containment system. Since a single manipulator would be unable to achieve the performance requirements at an acceptable cost, current APACTS design uses a series of three manipulators to position the system about the drydock, reach along the hull, and maintain proper standoff and motion.

This report reviews several motion trajectories to identify the effects of system criteria and to guide the APACTS manipulator system design. The motion-trajectory defines the relative motions between the manipulators and between the manipulators and the task. When combined with the performance requirements, the relative motions set the size and complexity of the manipulator, the manipulator's impact on other systems, and manipulator's effect on the quality of final paint coat.

\section{Methods, Assumptions, and Procedures}

This report reviews manipulators and identifies and evaluates trajectories with regard to known and assumed requirements of the APACTS system. Previous reports evaluate available manipulators based on the manipulator's ability to access areas of the ship's hull. This report extends the evaluation criteria to include APACTS' desired production rate, the Navy's coating system process rate, the required application accuracy, and known mechanical limitations of potential equipment. Evaluations are organized by trajectory. 
A trajectory is the sequence of points traversed by a manipulator to accomplish a task. The trajectory, the task, and the manipulator are inter-related. That is, a change in the task or a change in the manipulator forces a change in the trajectory and vice-versa. APACTS requires a manipulator and trajectory that will effectively, reliably, and affordably apply Navy coatings to Navy ship hulls.

\subsection{APACTS Manipulator Overview}

On March 9, 1998, Battelle, of Columbus, Ohio, presented an initial review of systems and components for a large-volume painter. Battelle stated that suitable Placement/Positioning Systems are available for APACTS and further development in this area is not warranted. Battelle identified five companies as major candidates to be manipulator suppliers; Pratt\&Whitney Water Jet Systems (WJS), PaR Robotics, CAE, Flow International, and AutoCrawler. Battelle's findings were based on minimal performance requirements of hull access (greater than $80 \%$ of area with greater then $1.8 \mathrm{~m}$ (72 in) radius of curvature). Additional requirements for economy and effectiveness necessitate reconsideration of the initial evaluation.

Paints are applied wet and require time to dry and cure. Mechanical contact during the cure time damages the coating. Therefore, for devices that rely on contact (e.g., surface crawlers), any contact behind the paint line (i.e., on the wet paint) is strictly forbidden. Surface crawler designs must include a priori paint schedules with crawler positions and orientations to allow full curing before application of subsequent paint coats. To avoid incidental contact with the wet paint and to maintain maximum scheduling flexibility, CDNWSC forbids any contact between APACTS and the hull surface [1].

APACTS carries the containment shroud along and just above the surface with sufficient accuracy to maintain the proper standoff for paint application and overspray containment [1]. The actual standoff value and its tolerance will depend largely on the responsiveness of the vacuum recovery system currently under study. The current shroud design requires a $2.5 \mathrm{~cm}$ (1 in) standoff, has an unknown tolerance, and removes air at the rate of 0.42 $\mathrm{m}^{3} / \mathrm{s}(900 \mathrm{cfm})$ to maintain the containment.

The APACTS system must maintain production and process rates. The production rate is the size of the painted area divided by the time required to paint that area. The process rate is the nozzle speed required to apply paint at the proper thickness. Since the production rate is less then the process rate, the paint gun can be stopped and relocated to an adjacent paint area and still achieve the overall production goal. The time available for the relocation depends on the relationship between the spraying and relocation times. In general, the relocation must be fast and accurate or the paint-on area must be large.

For a single manipulator, accuracy, volume, and speed are competing goals (i.e., accuracy improvements are achieved at the expense of speed or volume). APACTS will use a set of 
three, serially connected manipulators: a base, a maxi-manipulator, and a minimanipulator. The base fulfills the large volume requirement by moving the entire system to locations around the hull. The maxi-manipulator has limited speed and accuracy but sufficient reach to fulfill the access requirement. The mini-manipulator, to which the paint nozzle and shroud are attached, has far less reach but enough responsiveness and accuracy to compensate for the maxi-manipulator and to fulfill the system's performance requirements.

The base, maxi-manipulator, and mini-manipulator model is very flexible and may be applied to many motion devices, including hull crawlers, tracked systems, and actuated arms. The hull crawler's base is the safety line and power unit, its maxi-manipulator is the crawling device, and the mini-manipulator is the arm that holds the containment shroud over the surface. Tracked systems have large tracks erected about the hull as their maximanipulator. The mini-manipulators travel along these tracks and carry the paint nozzles and shroud to the surface. The tracks can carry substantial loads with excellent accuracy. However they are not truly portable and are intrusive to other dry-dock activities. The tracked systems have, therefore, been deemed unacceptable for APACTS. Actuated arms are generally based on commercial aerial work platforms (i.e., boomlifts, jlg's, etc.). Since crawlers and tracked systems are unacceptable for APACTS, actuated arms are the basis of the discussions throughout the remainder of the report. Other motion systems that may be discovered during the project can be evaluated using the same criteria.

Commercial aerial work platforms are long actuated arms on mobile bases and can be considered as a relatively inexpensive combination of two APACTS manipulators. The work platform's arm is equipped with sensors, servo controllers, and a supervisory controller to permit simultaneous coordinated motions of actuators and to coordinate actions with the mini-manipulator. Coordinated motion enables the maxi-manipulator to follow the general contours of the hull surface. Coordination with the mini-manipulator permits higher accuracy than possible with the aerial work platform alone.

Aerial work platforms are sluggish devices meant to carry workers to generally inaccessible areas. To the lift manufacturer, industry organizations, and government regulators, safety is a greater concern than speed or accuracy. The control valves use closed center spools that produce a broad stop region between the motion directions. The actuators incorporate holding valves to prevent rapid actuator movement in the event of power loss or operator error. These features protect workers on and near the work platform but limit the system responsiveness.

Due to its large mass and the low bandwidth actuators, an aerial work platform has difficulty initiating small, quick motions. Around zero velocity, the slow response, the closed center valves, and the holding valves produce deadbands, hysteresis, and other non-linear motion responses. However, when the spool is off center and the holding 
valves are open, the motion response is fairly linear. Therefore a commercial aerial work platform can provide clean motions on long, continuous trajectories.

The commercial work platform has four degrees-of-freedom in five actuated joints. The degrees-of-freedom provide a 3D location $(X, Y, Z)$ and rotation about the vertical axis. The fifth actuated joint maintains the vertical orientation of the final joint.

One APACTS concept paints with broad stripes that require five degrees-of-freedom: the $3 \mathrm{D}$ location, rotation about the painting axis, and rotation about the axis that is perpendicular to the paint stripe and parallel to the surface. The maxi-manipulator provides the first four degrees-of-freedom. The mini-manipulator provides the final degree of freedom and, by rapidly adjusting the standoff, fulfills the system's accuracy requirements. Thus, at a minimum, the mini-manipulator has two degrees-of-freedom. The mini-manipulator may incorporate additional degrees-of-freedom to move the paint sprayer along the surface.

\subsection{Trajectory Alternatives}

The path of the sprayer's motion is called the trajectory. It consists of the noncompensating motions of the mini-manipulator, the motions of the maxi-manipulator, and their interactions. Many paths can lay the proper amount of paint on a surface and numerous devices can follow those paths. The selection criteria for the APACTS manipulator will include the quality of the coating and the cost of the apparatus. The cost

will be a function of the size, weight, and complexity of the device and its impact on other APACTS components. The paint coat quality derives from the ability to maintain the application parameters and the ability to control the paint overlap.

This report compares four possible trajectories. The most basic trajectory uses intermittent motion of the maxi-manipulator and a completely independent minimanipulator. The second trajectory requires a very slow spray gun to paint at the maximanipulator's surface following speed. The third trajectory uses a very fine atomizing nozzle to spray multiple, thin layers as the maxi-manipulator maintains a constant speed along the hull contour. The last trajectory has the mini-manipulator compensate for the maxi-manipulator motion to produce paint bands perpendicular to the paint stripe.

\subsection{Evaluations}

Three values are computed for comparison of the trajectories. The access area reflects the size and the complexity of the mini-manipulator. The capture and treatment capacity is the amount of air that must be purified and reflects the burden that the trajectory places on other APACTS systems. The relative coating thickness shows the effect of the trajectory on the coating and reflects the quality of the final product. Other advantages and disadvantages, that are unique to the particular trajectory, are also discussed.

Review of Trajectories for the Automated Paint Application, Containment, and Treatment System 


\subsubsection{Access Area}

The mini-manipulator's access area implies its size, weight, and complexity. The access area is computed based on the effects of parameters such as paint gun speed, paint gun coverage, maxi-manipulator speed, and APACTS production rate. The resultant effects on weight and complexity are implied but are not computed. The mini-manipulator is always more complex than the access area since the mini-manipulator must also compensate for maxi-manipulator inaccuracies and provide the standoff and the horizontal rotations.

The access area is the length and width of the rectangular zone the mini-manipulator must reach along the surface. The rectangular zone must have sufficient depth (i.e., along the surface perpendicular) to accommodate the maxi-manipulator compensation. The access area includes the paint area plus some surrounding buffer sized to compensate for alignment error and for nozzle/shroud acceleration and deceleration.

For comparisons, acceleration based buffers are the length of a constant acceleration from zero to the nominal paint speed. Thus the acceleration/deceleration buffers are equal to one half the nominal paint speed times the square of the allotted time. Since one second is allotted for acceleration (see Table 1), the buffer is numerically equal to the nominal paint speed for the trajectory. This value is only for comparisons, as future experiments may indicate that different acceleration techniques with a larger or smaller buffer are required.

Table 1. Comparison Values

\begin{tabular}{ll} 
Symbol & \multicolumn{1}{c}{ Value } \\
$\mathrm{W}$ & $61 \mathrm{~cm}(24 \mathrm{in})$ \\
$\mathrm{W}_{\mathrm{e}}$ & $46 \mathrm{~cm}(18 \mathrm{in})$ \\
$\mathrm{G}_{5}$ & $20 \mathrm{~cm} / \mathrm{s}(8 \mathrm{in} / \mathrm{s})$ \\
$\mathrm{P}$ & $650 \mathrm{~cm}^{2} / \mathrm{s}\left(100 \mathrm{in}^{2} / \mathrm{s}\right)$ \\
$\mathrm{t}_{\text {Acceleration }}$ & $1 \mathrm{~s}$ \\
$\mathrm{M}$ & $5 \mathrm{~cm} / \mathrm{s}(2 \mathrm{in} / \mathrm{s})$
\end{tabular}

\section{Meaning}

Spray Width

Effective Spray Width

Nominal Paint Gun Speed

Desired Production Rate

Acceleration Time

Maxi-manipulator Speed

The paint area is an algebraic computation based on the need to cover an area in a proportional amount of time. The computation combines the desired overall production rate and the productivity of the paint gun. Unfortunately, the component values of gun productivity are not known. In order to compare the trajectories, values are assigned to the components based on observations by painters.

The general production rate is a quantified performance constraint. The Navy requires the overspray containment system be as productive as the current open spray system, estimated at $230 \mathrm{~m}^{2}\left(2500 \mathrm{ft}^{2}\right)$ per hour [1]. Since, the instantaneous productivity of the paint equipment is generally greater than the production goal, the production rate is attained through combinations of painting time and the non-painting time used to relocate 
or realign the application system. The $230 \mathrm{~m}^{2} / \mathrm{hr}$ value does not include the time to relocate to a different area of the hull (i.e., the base relocation time).

The process rate for the paint applicator is still being determined. Due to the need to apply high solids paints, the Navy has specified the airless spray gun for the initial versions of the APACTS [1]. Navy personnel feel the airless spray gun lays $0.13 \mathrm{~mm}$ $(0.005 \mathrm{inch})$ of paint at lateral speeds of $15 \mathrm{~cm} / \mathrm{s}$ to $30 \mathrm{~cm} / \mathrm{s}(6 \mathrm{in} / \mathrm{s}$ to $12 \mathrm{in} / \mathrm{s})$. The actual value depends on several factors including paint type and operating pressure. The width of the spray varies from $30 \mathrm{~cm}$ to $61 \mathrm{~cm}$ (12 in to $24 \mathrm{in})$ depending on the spray gun's nozzle and the distance between the nozzle and the surface. In addition the spray requires approximately $25 \%$ overlap. The inter-relationships between these parameters have not been quantified. The comparisons presented in this report use the values shown in Table 1. These values are reasonable, but not final.

\subsubsection{Containment System}

APACTS surrounds the paint spray with a shroud designed to contain, capture, and evacuate the paint overspray and free pollutants. The Gas Dynamics Laboratory (GDL) at Penn State University is developing the shroud. The shroud's size is primarily limited by the access requirement regarding radius of curvature and the size of the expectant paint spray. The maximum expected paint spray is $\pm 30 \mathrm{~cm} \mathrm{(} \pm 12$ in) from the center for the Navy's airless spray gun. The shroud is, therefore, shaped to collect a $30 \mathrm{~cm}$ overspray. A $1.8 \mathrm{~m}$ (72 in) radius of curvature produces a $2.5 \mathrm{~cm}$ (1 in) gap at $30 \mathrm{~cm}$ from the point of contact on a tangent. If this gap variation exceeds the tolerance of the shroud design, the shroud will be redesigned for alternate nozzles or guns as required.

GDL indicates the shape of a properly sized shroud produces a natural vortex that peels the overspray from the surface. Once caught in the vortex, the overspray is drawn off through a vacuum hose to remote purification equipment.

To ensure complete overspray capture, the vacuum maintains a slight inward airflow around the shroud perimeter. However most of the air withdrawn by the vacuum is entrained by the paint spray. Thus the vacuum requirement is a function of the number of nozzles, not the shroud size. As of March 1998, GDL calculated the flow requirement to be $0.47 \mathrm{~m} / \mathrm{s}(1000 \mathrm{cfm})$ per nozzle. These calculations are based on a $2 \mathrm{D}$, laboratory model and are subject to change. However, the number of nozzles required to achieve the production rate will greatly impact the size and cost of the collection and treatment equipment.

\subsubsection{Coating Thickness Estimation}

The Navy specifies $0.13 \mathrm{~mm} \pm 0.02 \mathrm{~mm}(0.005 \mathrm{inch} \pm 0.001 \mathrm{inch})$ of paint for each coat in a five-coat system [1]. The thickness is a function of the nozzle, paint type, and 
application parameters such as pressure, speed, and standoff. However, the trajectory may also affect the layer thickness variability due to the variable proximity of multiple path segments to a surface area. To appraise the trajectory's impact on thickness variability, paint spray distribution was measured and projected onto the trajectory path.

All trajectories affect paint thickness. Since the paint spray thins near the extremes, a painter overlaps the spray to produce a consistent layer. That is, the distance between the path segments is less than the width of the paint spray. The amount of paint at any point is the sum of the paint from each of the nearby path segments.

The trajectories proposed for APACTS are evaluated by summing the paint from each trajectory path segment, the maximum and minimum values are determined, and the percent difference computed and compared with the goal variation tolerance. The APACTS' goal is to apply between $0.10 \mathrm{~mm}$ and $0.15 \mathrm{~mm}(0.004$ inch to $0.006 \mathrm{inch})$ of paint with each coat, or to maintain a $33 \%$ variation in the thickness. The trajectory affects only the relative thickness. The painting parameters (speed, pressure, standoff, etc.) will be selected through experiments to produce the desired average paint thickness.

The paint thickness at any point is the sum of the paint sprayed on that point from each of the trajectory's path segments. The amount from a single path is a function of the distance from the surface point to the path segment along the major axis of the paint spray. The spray distribution is the function that determines the amount of paint and the distance from the center of the spray.

The airless paint gun's spray distribution is not known. For the comparisons, data was collected at CD-NSWC on a single "squirt" of paint from a conventional (i.e., air assisted) spray gun. Dry film thickness measurements were made with a dry film thickness gauge at $2.5 \mathrm{~cm}(1 \mathrm{in})$ intervals from the center of the spray and are shown in Figure 2. Six repetitions were made at each location and the median values used in the subsequent calculations. Paint at and beyond $20 \mathrm{~cm}(8 \mathrm{in})$ was too sporadic for measurements and a zero value was assigned. 
Figure 2. Paint Spray Distribution from Air Paint Gun

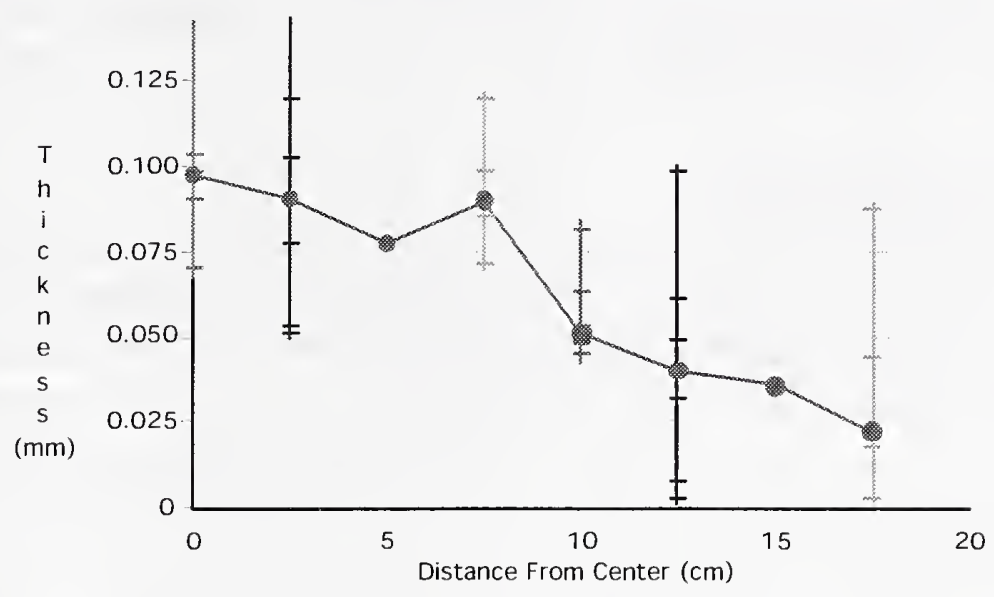

There are noticeable differences and similarities between the air sprayer squirt in Figure 2 and the anticipated distribution for an airless paint gun. Figure 2's vertical lines indicate the thickness variance is greater at each position than the APACTS tolerance allows. Furthermore, the spray width, at $40 \mathrm{~cm}$ (16 in), is far less than the $60 \mathrm{~cm}$ (24 in) anticipated for the airless sprayer. The assumption for the report's comparisons is only that the test spray distribution is similar to the airless spray distribution. For example, the $10 \mathrm{~cm}$ (4 in) spray overlap is proportional to the overlap expected for the airless paint gun. The report's trajectory comparisons are adjusted for the smaller spray width.

\section{Results and Discussions}

Four trajectories are considered for APACTS and are explained below. The most appropriate solution depends on restrictions that have yet to be quantified. The trajectories are available for all types of motion systems. However, since size and speed limits for hull crawlers are unknown and the Navy desires no hull contact, comparisons are based on the movements of the actuated arms.

\subsection{Intermittent Movement}

Under the intermittent movement approach, the maxi-manipulator carries the minimanipulator into position above a target area. The mini-manipulator paints the area while the maxi-manipulator remains stationary. The maxi-manipulator then carries the minimanipulator to the next area and the cycle repeats. The advantage of this approach is its simplicity. However the approach generates large non-painting periods during the maximanipulator relocation, which requires a large mini-manipulator to achieve the production rate.

Conventional manipulators can act as the maxi-manipulator for intermittent movement as currently manufactured. A mini-manipulator would be mounted on a standard boomlift Review of Trajectories for the Automated Paint Application, Containment, and Treatment System 
(the maxi-manipulator) and positioned near the ship hull. The positioning capabilities of the standard lift are limited. Therefore, the mini-manipulator would have to compensate for standoff, alignment, and overlap errors.

\subsubsection{Access Area Dimensions}

The size of the mini-manipulator operating area compensates for the lost productivity during shroud relocation. Since the gun speed to lay the $0.13 \mathrm{~mm}(0.005 \mathrm{inch})$ of paint $\left(G_{5}\right)$ times the width of the paint spray $\left(W_{\mathrm{e}}\right)$ is greater then the total production rate $(\mathrm{P})$, an oversized area can compensate for the relocation time.

The total production rate is the mini-manipulator's access area divided by the production time, or the time to paint one section.

$$
P=L_{\text {Paintarea }} H_{\text {Paintarea }} / \tau_{\text {Production }} \quad \text { EQ } 1
$$

The production time is the sum of the mini-manipulator relocation time, the nozzle-on time, and the time required to accelerate the nozzle. The nozzle-on time is the product of the time to paint one stripe and the number of stripes in the access area. The acceleration time is the time to bring the nozzle from rest to the application speed times the number of accelerations and decelerations, that is one less then twice the number of stripes.

$$
\tau_{\text {Production }}=\tau_{\text {Relocation }}+\frac{L_{\text {Paintarea }}}{G_{5}} \frac{H_{\text {Paintarea }}}{W_{e}}+\left(2 \frac{H_{\text {Paintarea }}}{W_{e}}-1\right) \tau_{\text {Acceleration }} \text { EQ } 2
$$

Relocation time is a function of the travel speed, the distance traveled, and the time to realign the shroud to the task. Experiments conducted in 1993 for the Navy's Paint Stripping Program developed expected values for relocation speed and alignment. Reliable speeds were around $20 \mathrm{~cm} / \mathrm{s}(8 \mathrm{in} / \mathrm{s})$ and alignment times varied from 15 seconds, for loose tolerances, to 100 seconds for tight tolerances.

$$
\tau_{\text {Relocation }}=\tau_{\text {Alignment }}+\frac{H_{\text {Paintarea }}}{S p d_{\text {Relocation }}}
$$

The required paint stripe length is found by combining equations 1 through 3 .

$$
L_{\text {PaintArea }}=\frac{P\left(\tau_{\text {Alignment }}+\frac{H_{\text {PaintArea }}}{S p d_{\text {Relocation }}}+\left(2 \frac{H_{\text {PaintArea }}}{W_{e}}-1\right) \tau_{\text {Acceleration }}\right)}{H_{\text {PaintArea }}\left(1-P /\left(G_{5} \times W_{e}\right)\right)} \quad \text { EQ } 4
$$

With the values from Table 1, the mini-manipulator paints five stripes in a $3.25 \mathrm{~m}$ by $2.29 \mathrm{~m}$ (128 in x $90 \mathrm{in})$ area. The access buffer is $20 \mathrm{~cm}(8 \mathrm{in})$ for acceleration and $41 \mathrm{~cm}$ (16 in) for alignment. Thus the mini-manipulator's required access area is roughly $3.9 \mathrm{~m}$ by $2.7 \mathrm{~m} \mathrm{(150} \mathrm{in} \mathrm{x} 106 \mathrm{in})$.

Review of Trajectories for the Automated Paint Application, Containment, and Treatment System 
Unfortunately a $3.9 \mathrm{~m}$ by $2.7 \mathrm{~m}$ mini-manipulator exceeds the carrying capacity of commercial manipulators. The Hull-Jet by Pratt\&Whitney Water Jet Systems has a $1.8 \mathrm{~m}$

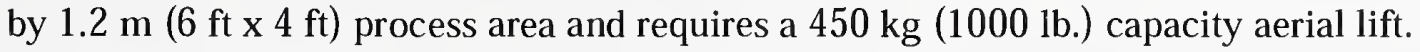
Thus a lift with approximately $2200 \mathrm{~kg}(4900 \mathrm{lb}$.) capacity would be required for this approach to APACTS. Since industry ceased production of manipulators with greater then $340 \mathrm{~kg}(750 \mathrm{lb}$.) capacity in 1997, special built hardware must be designed and built.

Equations 1 through 4 indicate larger spray widths or faster gun speeds allow similar production rates with smaller manipulators. For example, a system with two nozzles would paint two $91 \mathrm{~cm}$ (36 in) wide stripes in a $2.1 \mathrm{~m}$ by $2.4 \mathrm{~m}(7 \mathrm{ft} \times 8 \mathrm{ft})$ area, which still exceeds the commercial size limits.

A system with three nozzles could paint a $140 \mathrm{~cm}$ (54 in) stripe in a single pass, eliminating the requirement to reverse the gun direction. The required stripe length would be $1.4 \mathrm{~m}(55 \mathrm{in})$ and the access area would be $2.0 \mathrm{~m}$ by $1.8 \mathrm{~m}$ ( 79 in $\mathrm{x} 70 \mathrm{in})$. Although this access area still exceeds the Hull-Jet dimensions, no motion is required in the vertical direction and construction of a mini-manipulator within the lift capacity of commercial machines may be possible. The triple nozzle approach may pose additional problems with the relocation time. Since there is no vertical motion that can correct for misalignments, the maxi-manipulator's position must be far more accurate, which increases the relocation time. For any of these solutions, if the relocation time proves greater than the minimum, the mini-manipulator will be significantly larger.

\subsubsection{Capture and Treatment Capacity}

The capture and treatment capacity computation is the same for all of the trajectories. The capacity is a function of the number of paint nozzles in the application system.

$$
\text { Airflow }=0.42 \times \text { Nozzles } \quad \text { EQ } 5
$$

More complex evaluations of the treatment capacity and shroud size are expected as the shroud technology advances.

Since the treatment capacity is a function of the number of spray nozzles, the first version reviewed above with a single nozzle requires $0.42 \mathrm{~m} / \mathrm{s}(900 \mathrm{cfm})$ to be treated. The three-nozzle system requires a $1.26 \mathrm{~m}^{3} / \mathrm{s}(2700 \mathrm{cfm})$ treatment. The flow and treatment requirements are subject to change as the shroud technology develops.

\subsubsection{Paint Thickness}

Paint thickness variation attributable to the trajectory is a function of the overlap. For the case of parallel spray strokes, the overlap on the surface is the same as the overlap of the distribution. For the distribution in Figure 1, the overlap is $10 \mathrm{~cm} \mathrm{(4} \mathrm{in)} \mathrm{or} 25 \%$ of the spray width. The sum of two distributions, overlapped by $10 \mathrm{~cm}$, yields a relative Review of Trajectories for the Automated Paint Application, Containment, and Treatment System 
thickness between 3.0 and 3.9. These values have a $24 \%$ range and satisfy the APACTS goal of $33 \%$.

\subsection{Perpendicular Nozzles}

This trajectory overlaps several paint nozzles aligned perpendicular to the motion of the maxi-manipulator. This approach greatly simplifies the design of the mini-manipulator. The maxi-manipulator follows the general contour of the ship hull and maintains perpendicularity with the hull orthogonal to the maxi-manipulator's motion while the mini-manipulator maintains proper standoff and transverse perpendicularity. The approach alleviates problems associated with hull protrusions but requires new paint gun technologies and a larger containment shroud.

\subsubsection{Access Area Dimensions}

The paint length is a multiple of the effective spray width and the quotient of the desired production rate and the maxi-manipulator speed. Since the desired production rate $(\mathrm{P})$ and the effective spray width $\left(\mathrm{W}_{\mathrm{e}}\right)$ are quasi-fixed, the maxi-manipulator speed $(\mathrm{M})$ is a function of the number of guns (n).

$$
\begin{array}{ll}
L_{\text {PaintArea }}=n W_{e}=P / M & \text { EQ } 6 \\
M=\frac{P}{n W_{e}} & \text { EQ 7 }
\end{array}
$$

Equation 7 indicates reasonable maxi-manipulator speeds are derived with either two (7.1 $\mathrm{cm} / \mathrm{s}, 2.8 \mathrm{in} / \mathrm{s})$ or three $(4.8 \mathrm{~cm} / \mathrm{s}, 1.9 \mathrm{in} / \mathrm{s})$ paint guns. However both speeds are below the assumed gun speed for a $0.13 \mathrm{~mm}(0.005 \mathrm{inch})$ coating. Paint guns that can lay the proper amount of paint at these reduced speeds need to be identified or developed before using this approach.

\subsubsection{Capture and Treatment Capacity}

The containment shroud's size is a function only of the number of nozzles in the application system. A review of the computation is provided in section 4.1.2.

The trajectory requires two nozzles in the application system. Thus, in accordance with equation 5 , a treatment capacity of a $0.84 \mathrm{~m} / \mathrm{s}(1800 \mathrm{cfm})$ is required.

\subsubsection{Paint Thickness}

The portion of the paint thickness variation that is attributable to the trajectory is a function of the overlap. This trajectory, like the intermittent motion trajectory, paints Review of Trajectories for the Automated Paint Application, Containment, and Treatment System 
with parallel strokes. Parallel spray strokes overlap on the surface equivalent to the overlap of the distribution. For the relative distribution (i.e., dimensionless) in Figure 1, the overlap is $10 \mathrm{~cm}$ ( $4 \mathrm{in}$ ) or $25 \%$ of the spray width. The sum of two distributions, overlapped by $10 \mathrm{~cm}$, yields a 3.0 minimum relative thickness and a 3.9 maximum relative thickness. These values equal a $24 \%$ range and exceed APACTS' goal of $33 \%$.

\subsubsection{Other Considerations}

The Perpendicular Nozzle trajectory approach requires new paint gun and new nozzle technologies or vastly improved maxi-manipulator control. High-speed maxi-manipulator motions require higher accelerations that have induced oscillations in previous efforts. A paint gun that can reliably lay $0.13 \mathrm{~mm}(0.005 \mathrm{inch})$ of high solids paint at $5.0 \mathrm{~cm} / \mathrm{s}$ to 7.6 $\mathrm{cm} / \mathrm{s}$ ( $2 \mathrm{in} / \mathrm{s}$ to $3 \mathrm{in} / \mathrm{s}$ ) without clogging is also required. Perhaps a combination of nozzle, pressure, and standoff exists that can provide this performance. Further investigation into paint gun performance and improvements to the maxi-manipulator control are necessary to exploit the multiple perpendicular nozzle approach.

The multiple perpendicular nozzle mini-manipulator requires only two degrees-offreedom. The standoff control is a linear motion and the transverse perpendicularity control is a rotary motion. The ranges for these motions will be design dependent but are anticipated to be $15 \mathrm{~cm}$ ( $6 \mathrm{in}$ ) for the standoff control and roughly $90^{\circ}$ for the perpendicularity control. The absence of long linear motions reduces the hose complexities, reduces requirements for cantilevered loads, and reduces the overall system weight.

A secondary advantage of this approach is that the impacts of the containment shroud skirt with hull protrusions have less energy. Ships have numerous $9 \mathrm{~cm}$ (3.5 in) high padeyes welded to the hull. The containment shroud is envisioned to have a flexible skirt to allow the padeyes to pass under the shroud. Impact between the skirt and the padeyes at high speed may induce oscillations in the mechanically "soft" maxi-manipulator. The lower speed envisioned with this approach reduces the impact energy and thus reduces the oscillations that may result.

\subsection{Zigzag}

The zigzag trajectory relies on blending multiple high speed passes to achieve proper coating of the hull surface. There are two significant advantages to the zigzag trajectory; the natural blending of very thin layers of paint and a simple mechanical device. The disadvantages, or additional challenges, include fine atomization of the paint, achieving high lateral speeds without inducing oscillations, and handling potential impacts with hull protrusions at high speeds. 
The zigzag trajectory consists of repetitive cycles of horizontal paint gun motions by the mini-manipulator while the maxi-manipulator moves the mini-manipulator vertically. As shown in Figure 3, the combined motion forms a zigzag patern. The horizontal movement begins outside the access area, accelerates to reach the desired speed the edge of the access area where the paint spray is turned on. The paint is sprayed as the gun traverses the access area and stopped when the gun reaches the far edge. The gun then slows to a stop and the cycle repeats.

Figure 3. Zigzag Trajectory

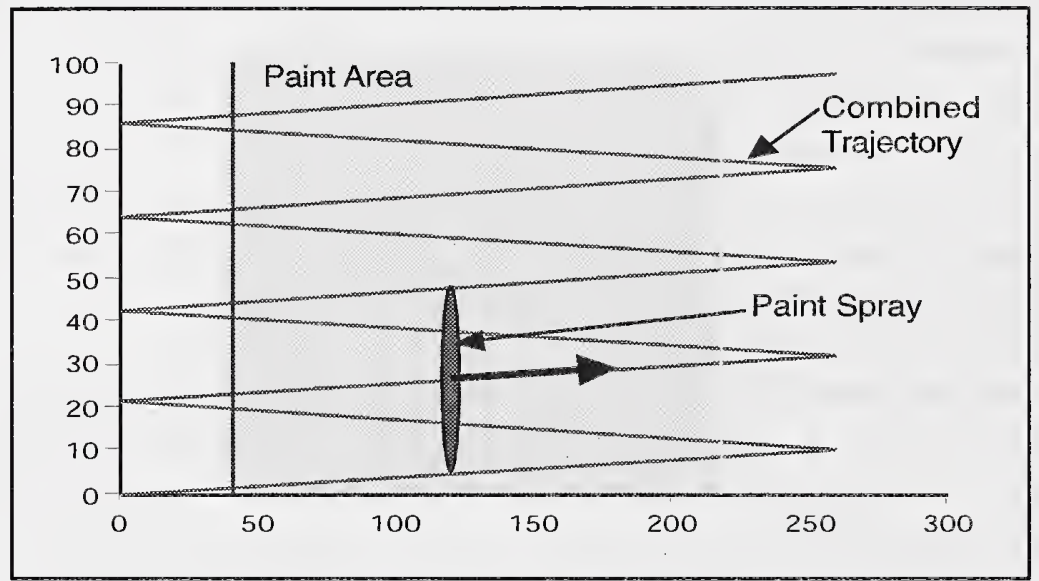

The trajectory makes several passes over the surface as the maxi-manipulator moves at a constant speed. The mini-manipulator moves the paint gun fast enough to complete a horizontal cycle before the maxi-manipulator has moved some fraction of the width of the paint spray (e.g., 1/4). Thus the spray will pass each surface point the inverse of that fraction number of times (e.g., 4 times). Each pass exposes the surface point to a different area of the spray distribution making a more even blend.

\subsubsection{Access Area Dimensions}

The dimensions and performance characteristics of the zigzag trajectory are tightly coupled with the performance characteristics of the paint gun.

The time for one horizontal pass is a function of the spray width (W) and the maximanipulator speed $(\mathrm{M})$ :

$$
\tau_{\text {Cycle }}=\frac{1}{r} \frac{W}{M}
$$

EQ 8

where $\mathbf{r}$ is the number of repetitions over the surface. Since the overlap is implicit in the zigzag trajectory, equation 8 uses the actual paint spray width rather than the effective spray width. The time over the paint area is the ratio of the horizontal length of the area and the speed of the paint gun. Thus the duration when the paint is spraying is:

Review of Trajectories for the Automated Paint Application, Containment, and Treatment System 


$$
\tau_{\text {Paint }}=\frac{L_{\text {Paintarea }}}{G_{5 / r}}
$$

The gun speed $\left(\mathrm{G}_{5 / \mathrm{r}}\right)$ is assumed to be the number of repetitions $(r)$ times the nominal gun speed $\left(G_{5}\right)$. The actual gun speed must be verified by experiment.

The time to slow and reverse the paint gun is the difference between the total cycle time and the time spraying. The time to accelerate and decelerate are assumed to be the same, thus:

$$
2 \tau_{\text {Accelerate }}=\tau_{\text {Cycle }}-\tau_{\text {Paint }} \quad \text { EQ } 10
$$

Finally, as with all continuous motion approaches, the length of the paint stripe is the quotient of the desired production rate $(\mathrm{P})$ and the maxi-manipulator speed $(\mathrm{M})$.

$$
L_{\text {Paintarea }}=P / M \quad \text { EQ } 11
$$

Combining equations 8 through 11 yields an equation for the maxi-manipulator speed as a function of the paint gun parameters $\left(\mathrm{W}\right.$ and $\left.\mathrm{G}_{5}\right)$, and the turnaround time:

$$
M=\left(W-\frac{P}{G_{5}}\right) /\left(2 r \tau_{\text {Acceleration }}\right) \quad \text { EQ } 12
$$

With the Table 1 values and four repetitions per layer, the paint stripe is $189 \mathrm{~cm}$ (70 in) long and the maxi-manipulator speed is $3.6 \mathrm{~cm} / \mathrm{s}(1.4 \mathrm{in} / \mathrm{s})$. The higher gun speed requires $81 \mathrm{~cm}$ (32 in) for acceleration and deceleration. Thus the mini-manipulator access area would be $259 \mathrm{~cm}$ (102 in) long but have no vertical component.

\subsubsection{Capture and Treatment Capacity}

The containment shroud's size is a function only of the number of nozzles in the application system. A review of the computation is provided in section 4.1.2.

The zigzag trajectory requires a single nozzle in the application system. Thus, in accordance with equation 5 , a treatment capacity of a $0.42 \mathrm{~m}^{3} / \mathrm{s}(900 \mathrm{cfm})$ is required.

\subsubsection{Paint Thickness}

While most trajectories lay a thick coat of paint with overlap along the edges, the zigzag trajectory lays many thin coats of paint, effectively overlapping throughout the painted area. The many small coats blend the irregularities in the paint spray and will diminish defects caused by errant oscillations in the system.

Figure 4 is the output of a simulation using the zigzag trajectory. The simulation computes the vertical distance from a point on the surface to each trajectory segment. The spray Review of Trajectories for the Automated Paint Application, Containment, and Treatment System 
distribution data in Figure 2 determines the amount of paint deposited on the surface point from the trajectory segment. The total paint at the surface point is the sum from all of the trajectory segments. In Figure 4, the side areas are the unpainted acceleration and deceleration zones and the top and bottom areas reflect the thinner paint caused by fewer passes as the trajectory starts and stops. Within the central region, the relative thickness is between 2.4 and 2.9. These values differ by about $17 \%$, well within the $33 \%(0.10$ $\mathrm{mm}$ to $0.15 \mathrm{~mm}, 0.004$ inch to $0.006 \mathrm{inch}$ ) tolerance specified for APACTS.

\section{Figure 4. Zigzag Trajectory Paint Distribution}

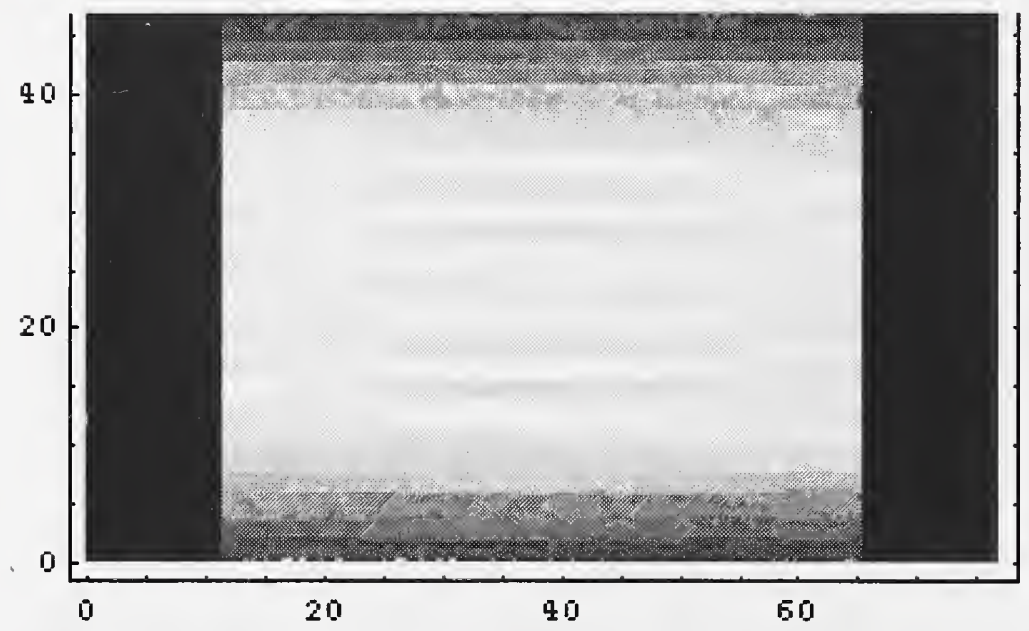

\subsubsection{Other Considerations}

Another trajectory measure is tolerance for oscillations along the paint axis. Figure 5 superimposes a $2.5 \mathrm{~cm}$ ( $1 \mathrm{in})$ amplitude, one hertz oscillation noise signal on the zigzag trajectory. In Figure 5, the relative thickness ranges from 2.2 to 3.3, or $34 \%$. This range is close to the APACTS performance specification and may have been achieved with a more ideal spray distribution. 
Figure 5. Zigzag Trajectory Paint Distribution with Noise

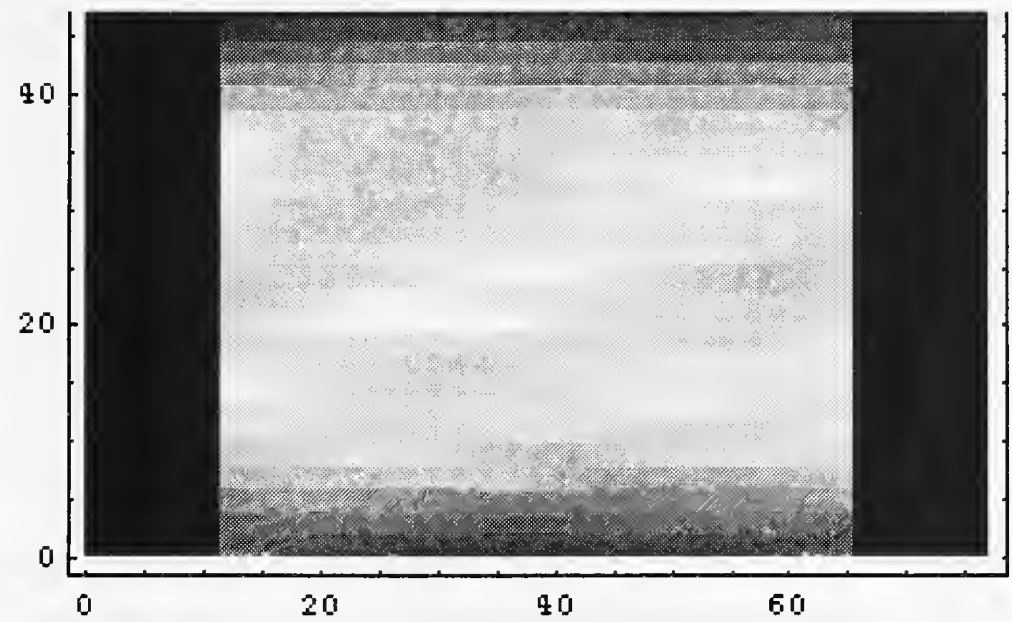

Neither the noise free simulation nor the simulation with the $1 \mathrm{~Hz}$ oscillation produces a $0.13 \mathrm{~mm}(0.005 \mathrm{inch})$ thick paint coat. This is the result of the comparison data not being normalized to an idealized spray and does not indicate a problem with the trajectory. For the actual APACTS, nozzles and pressures will be selected to produce the desired nominal thickness. Here, only the shape of the distribution is used in the comparison.

\subsection{Vertical Motion Compensation}

\subsubsection{Trajectory Description}

This approach paints horizontal stripes on the ship as the maxi-manipulator moves at a constant vertical speed along the surface. The mini-manipulator trajectory adds the desired horizontal motion to a vertical motion that is equal and opposite to the maximanipulator motion.

Figure 6 shows the two relative motions of the paint head in unitless dimensions. Figure 6(a) shows the trajectory as executed by the mini-manipulator while Figure 6(b) shows the resultant path on the ship when combined with a constant maxi-manipulator motion (shown as $2 / \mathrm{s}$ ). For simplicity, the displayed trajectory uses a constant velocity circular turnaround. The APACTS application may require a more sophisticated trajectory including smooth accelerations in Cartesian space and along joint actuators. 
Figure 6. Head Trajectories; Relative to (a) Manipulator, (b) Ship

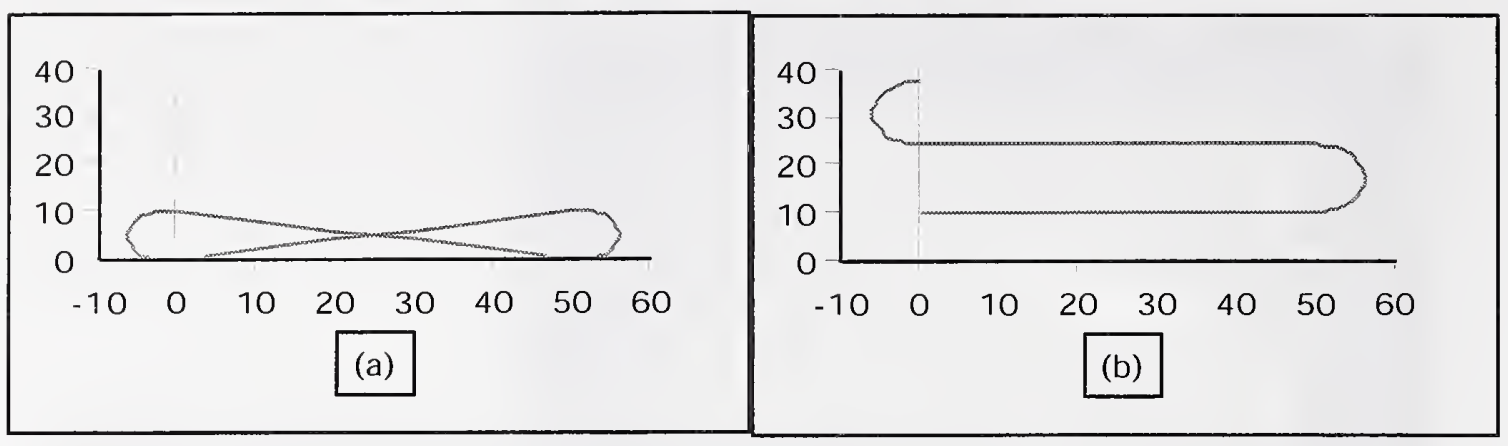

\subsubsection{Access Area Dimensions}

The production rate is the ratio of the painted area and the painting time. For the vertical motion compensation trajectory, the production rate is the area of the horizontal paint strip divided by the time to paint the stripe and the time required to accelerate to the appropriate paint speed and decelerate back to stop. The paint stripe area is the product of the paint area's horizontal length and the effective width of the paint spray.

$$
P=\frac{L_{P_{\text {aintArea }}} \times W_{e}}{\frac{L_{\text {PaintArea }}}{G_{5}}+2 \tau_{\text {Acceleration }}}
$$

The size of the paint area length is also a function of the maxi-manipulator speed.

$$
L_{\text {Paintarea }}=P / M
$$

EQ 14

The vertical component of the paint area (i.e., the height or $\mathrm{H}_{\text {PaintArea }}$ ) is the distance the maxi-manipulator moves while the paint head traverses the horizontal paint stripe.

$$
H_{\text {Paintarea }}=M \frac{L_{\text {Paintarea }}}{G_{5}}
$$

EQ 15

For the comparison values from Table 1, the paint area is $180 \mathrm{~cm}$ by $38 \mathrm{~cm}$ (70 in by 15 in) and 2.75 seconds would be available to reverse the direction of the paint gun. The total access area would be $200 \mathrm{~cm}$ by $43 \mathrm{~cm}$ (78 in by $17 \mathrm{in})$.

\subsubsection{Shroud Size}

The containment shroud's size is a function only of the number of nozzles in the application system. A review of the computation is provided in section 4.1.2.

The trajectory requires a single nozzle in the application system. Thus, in accordance with equation 5 , a treatment capacity of a $0.42 \mathrm{~m}^{3} / \mathrm{s}(900 \mathrm{cfm})$ is required. 


\subsubsection{Coating Thickness}

Paint thickness variation attributable to the trajectory is a function of the overlap. This trajectory, like the intermittent motion trajectory, paints with parallel strokes. Parallel spray strokes overlap on the surface equivalent to the overlap of the distribution. For the distribution in Figure 1, the overlap is four inches or $25 \%$ of the spray width. The sum of two distributions, overlapped by $10 \mathrm{~cm}(4 \mathrm{in})$, yields a minimum relative thickness of 3.0 and a maximum relative thickness of 3.9 . These values equal a $24 \%$ range that satisfies the APACTS goal of $33 \%$.

\section{Conclusions}

\subsection{Available Systems}

Battelle's March 9th review states that work on the manipulation system is unnecessary since existing solutions are available. Battelle's report identifies five existing manipulator systems to support the claim; Pratt\&Whitney Water Jet Systems, PaR Robotics, CAE, Flow International, and AutoCrawler. All five have sufficient operating volumes for APACTS, however none fulfill the known system requirements for speed, accuracy, and contact.

Flow International and AutoCrawler violate the non-contact requirement. Both are hull crawler systems developed for paint stripping. Hull crawler use requires the Navy to abandon the flexibility to add paint over less than fully-cured coats. If such flexibility is no longer required, the hull crawler's ability to carry and maneuver large cantilevered loads must be assessed.

Pratt\&Whitney's system uses a move-press-n-hold approach for paint stripping. A commercial boomlift carries a rectangular manipulator to a work area and presses the manipulator onto the surface. Pratt\&Whitney's approach is similar to the intermittent motion trajectories with surface contact. The required mechanical size to achieve specified production rates exceeds the carrying capacity of available boomlifts. Thus the Pratt\&Whitney approach is inadequate for APACTS.

The PaR system has not had a public demonstration. Discussions with PaR Robotics indicate that they use the intermittent movement approach described above. $\mathrm{PaR}$ uses a high-extension forklift that can carry greater loads than an aerial work platform. Thus the PaR system may be able to carry APACTS. However a forklift has only three degrees-offreedom. The base must be relocated far more frequently and the base positioning options are more restricted. A demonstration is required before the $\mathrm{PaR}$ system can be adequately evaluated.

The CAE system is a concept with drawings but few specifics. CAE appears to use large rails formed to match the contours of the target surface. These rails do not appear Review of Trajectories for the Automated Paint Application, Containment, and Treatment System 
portable with standard actuator devices. Setup and relocation time for the rails may take hours making required production rates impossible to achieve. Since the rails match the surface profiles, the cost to build, store, and maintain the rails must be included in an evaluation. The CAE approach is probably not suitable for the number of ship types to be handled by APACTS.

The Pratt\&Whitney, PaR, CAE, Flow, and AutoCrawler systems have sufficient operating volume to access the major portions of a ship hull. However, in addition to large volume, APACTS requires minimal or no contact, good accuracy, and speed. The applicability of a solution must be evaluated under all available criteria. Under these criteria, and with the available information, these five systems are inadequate.

\subsection{Trajectory Comparisons}

Table 2 summarizes the effects of the different trajectories with the performance requirements and specifications in Table 1 . The values are not absolute, but are the results of functions of the underlying assumptions. Ultimate trajectory selection and subsequent mini-manipulator design must await quantification of system parameters.

The results in Table 2 change with the base assumptions. For example, the intermittent move trajectory values are sensitive to the sprayer speed. A faster speed results in a smaller access area. However, the access area is likely to remain larger then the commercial lift capacity. Even when the spray gun's speed is infinite, the paint area $(1.4 \mathrm{~m}$ by $0.9 \mathrm{~m}$, 56 in by 36 in) remains significantly larger then the Hull Jet and would exceed commercial boomlift capacity.

Table 2. Comparison Summary

\begin{tabular}{|l|c|c|c|l|}
\hline \multicolumn{1}{|c|}{ Trajectory } & $\begin{array}{c}\text { Access } \\
\text { Area (cm) }\end{array}$ & $\begin{array}{c}\text { Treatment } \\
\text { Capacity }\end{array}$ & $\begin{array}{c}\text { Thickness } \\
\text { Range }\end{array}$ & \multicolumn{1}{|c|}{ Comment } \\
\hline Intermittent Move (1 Gun) & $390 \times 270$ & $0.47 \mathrm{~m}^{3} / \mathrm{s}$ & $23 \%$ & Excessive weight \\
\hline Intermittent Move (3 Gun) & $200 \times 41$ & $1.43 \mathrm{~m}^{3} / \mathrm{s}$ & $23 \%$ & \\
\hline Perpendicular Nozzles & $0 \times 0$ & $0.94 \mathrm{~m}^{3} / \mathrm{s}$ & $23 \%$ & Needs slower nozzles \\
\hline Zigzag & $260 \times 0$ & $0.47 \mathrm{~m}^{3} / \mathrm{s}$ & $17 \%$ & Needs fine spray nozzles \\
\hline Vertical Compensated & $200 \times 43$ & $0.47 \mathrm{~m}^{3} / \mathrm{s}$ & $23 \%$ & \\
\hline
\end{tabular}

The maxi-manipulator capabilities also impact the trajectory requirements. For example, if the maxi-manipulator resists induced oscillations, faster accelerations would permit smaller access areas, particularly for the zigzag trajectory. When the acceleration time is reduced to 0.7 seconds, the zigzag access area length reduces to about $2 \mathrm{~m}$ ( $80 \mathrm{in})$.

Trajectory selection should be based on quantified criteria. Data is required on the maximanipulator's speed along ship contours, on the permissible mini-manipulator

Review of Trajectories for the Automated Paint Application, Containment, and Treatment System 
accelerations that do not excite maxi-manipulator oscillations, and on the application system's spray width, spray distribution, and nozzle speed. When the data is available, the manipulator may be designed and the trajectory selected for an effective, economical, and environmentally sound paint system.

\section{Recommendations}

\subsection{System Review}

The manipulation system from PaR Robotics may be sufficient for APACTS. Before a final decision, APACTS technical and integration personnel should review the $\mathrm{PaR}$ system.

\subsection{Parameter Verification}

The values used for comparisons should be quantified. The trajectory and manipulator selection depends on paint gun speed, distribution, and atomization, and the maximanipulator's speed and tolerance to induced oscillations. The following experiments should be conducted:

- construct a rail mounted spray gun and measure the interrelationships of the nozzle, pressure, and paint type with the speed.

- measure maxi-manipulator response to typical ship contours at higher speeds.

- measure the effects of mini-manipulator motions to induce oscillations in the maximanipulator.

\subsection{Investigate Command/Control}

The maxi/mini concept proposed for APACTS is a departure from standard controls practice. Generally a manipulator receives world location data from tightly coupled sensors. For example, the maxi-manipulator would have a sensor to measure the distance to the surface. However, under the maxi/mini paradigm, the range to the surface is irrelevant. The maxi-manipulator must react to the needs of the mini-manipulator to stay within its motion range.

Controller response to the status of associated equipment is within the hierarchical control concept of NIST's Real-time Control System but has not been implemented on distributed equipment. Distributed control offers cost savings through reduced wiring and hardware but limits the amount of information that can flow between the various levels and nodes of a control system. While monolithic control, a single computer or single computer location, offers almost unlimited data transfer, the information flow requirements appear to be within the distributed control limits.

Review of Trajectories for the Automated Paint Application, Containment, and Treatment System 


\subsection{Component Coordination}

Coordination between the various APACTS component developers is required to ensure the performance requirements imposed by one component on another component does not exceed the physical limitations of the other component.

\section{References}

1. Carderock Division, Naval Surface Warfare Center, SOL N00167-97-SS-R1, "Mechanical Ship Hull Paint Application System For Use in Drydock", Commerce Business Daily, April 10, 1997.

\section{Symbols}

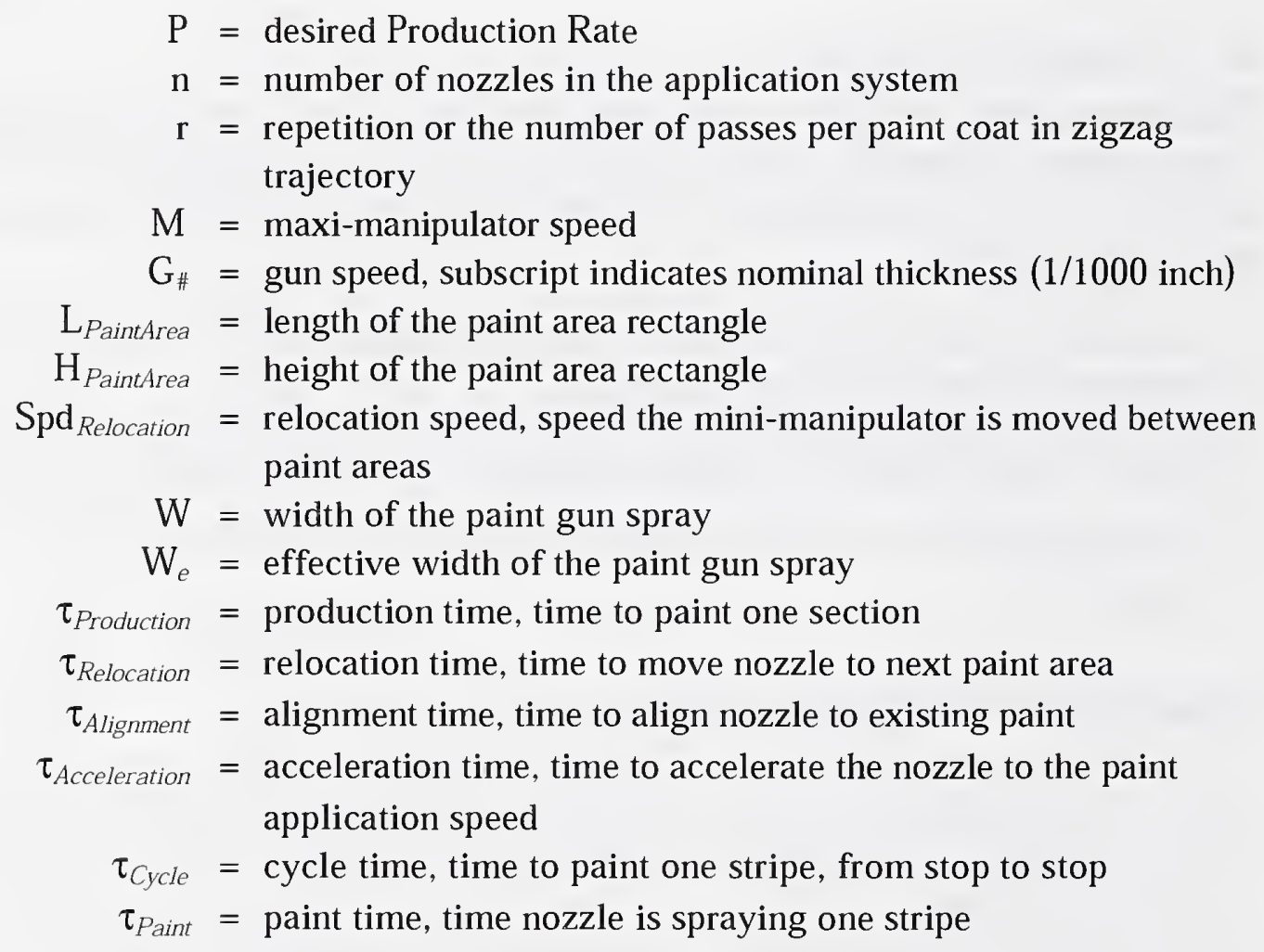




\section{.}


